\title{
Survey on - Socially Intelligent Robots by using NLP
}

\author{
Shruthi J. \\ Assistant Professor, \\ CSE BMSIT\&M, Bengaluru
}

\author{
Suma Swamy, PhD \\ Associate Professor, \\ CSE SMVIT, Bengaluru
}

\author{
Sarika C. G. \\ MTech CSE \\ BMSIT\&M, Bengaluru
}

\begin{abstract}
The design of natural interaction with social robots is highly complex process, given the huge design space of robots in terms of appearance and behaviour and the challenges arising when using face detection and speech recognition in the wild. More natural and highly autonomous interaction is necessary to faster trust and engagement and hence establishing a longterm social relationship between users and robots. NLP is used to-extract user's basic information, hobbies and interests for building a rich user profile. This presents the framework design to enable the development of social robotic applications by cross-disciplinary teams of programmers and interaction designers and advantages and dis-advantages of social robots.
\end{abstract}

\section{Keywords}

Human robot interaction, social robots, user modelling, user profile, interaction design framework, robot applications.

\section{INTRODUCTION}

Various technologies are growing all around the world, Robotics and NLP are one among them. Natural language and robotics are two major areas of Artificial Intelligence - the theory and development of computer systems able to perform tasks normally requiring human intelligence, such as speech recognition, visual perception, decision-making, and translation between languages.

The field of social robotics is still young, and although much research has focused on details of creating human like interactions for social robots. However, this is a crossdisciplinary process integrating technical knowledge of hardware and software, psychological knowledge of interaction dynamics, and domain-specific knowledge of the target application. It is expected that social robots tomorrow will be like personal computers today. In future, personal robots will be able to help people in their daily activities. Socially intelligent robots could have a significant positive impact on real-world challenges, such as helping elders to live independently at home longer, serving as learning companions for children and enhancing the learning experiences through play or with various interactions, serving a therapeutic role to help children with autism learn communication skills.

As robots progress from its traditional very controlled settings such as factories and laboratory environments and are deployed in homes and social contexts, the ability of robots to interact with humans. It explores the idea of using NLP as a tool to help social robots to learn from natural interpersonal interactions with users, learn user's personal information and preferences and using this information to build a user profile which can be used subsequently to customize the dialogue system.

Natural language as a communication medium for humans is an efficient means to make a technical system easier accessible to its users. The main goal of natural language processing is to give a computer with the ability to interact with people the way people interact with each other. The objective of the social robotics research is to design robots that engage in social scenarios which are compelling and familiar to humans. Thus, the robots have to provide a social communicative functionality that is natural and intuitive. This can be supported if appearance and functionality fit the robots' tasks and robots are as self- explaining as possible. All these are very important while designing robots to interact with users in domestic environments.

Such a design process is inherently inefficient. To improve the efficiency in design of social robotics applications, a structured framework is necessary to enable these fundamentally distinct aspects of social robot application development to be conducted in parallel.

In this paper we propose a framework which uses clearlydefined layers of abstraction to allow this kind of parallel development. In this framework, programming specialists are free to focus on low-level programming tasks like hardware interfacing or data processing. These low-level components are then encapsulated and presented to interact with designers via an easy-to-use graphical interface for developing interaction flows and fine-tuning details of the robot's utterances and gestures.

\section{MODELLING TECHNIQUES}

\subsection{NLP for User Modelling}

NLP is used to help users to communicate with computers and robots which is important for casual users and those users who have no time to learn new interaction skills such children and elderly people. Subdivision of human computer interaction that describes the process of building up and modifying a conceptual understanding of the user. A user model is the collection and categorization of personal data which is associated with a specific user. It can include personal information such as users' names and ages, their interests, their skills and knowledge, their goals and plans, their preferences and their dislikes or data about their behaviour and their interactions with the system. There are different types of user models.

Static- where information can't be changed once it is updated.

Dynamic- which allows for more updation of users whenever changes in their interests, learning progress or interactions occurs.

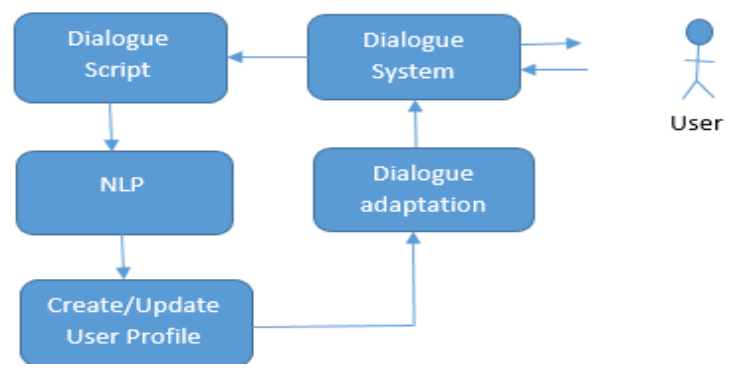

Figure1: Using NLP for Dynamic User Modeling. 


\subsection{User Modelling for Long-term Interaction}

Dialogue system is designed to collect the personal data associated with the user such as names, age, profession, country, city and town, likes and dis-likes, hobbies and interests and store it in a user profile. Fig1 shows that user profile is updated continuously whenever changes are detected When the robot starts a dialogue with a new user, it uses face detection to retrieve its user profile and use the information stored in that profile to customize the dialogue to give the user the feeling that the robot memories and care about him/her. If it fails to retrieve the user profile, then it will create a new user profile.

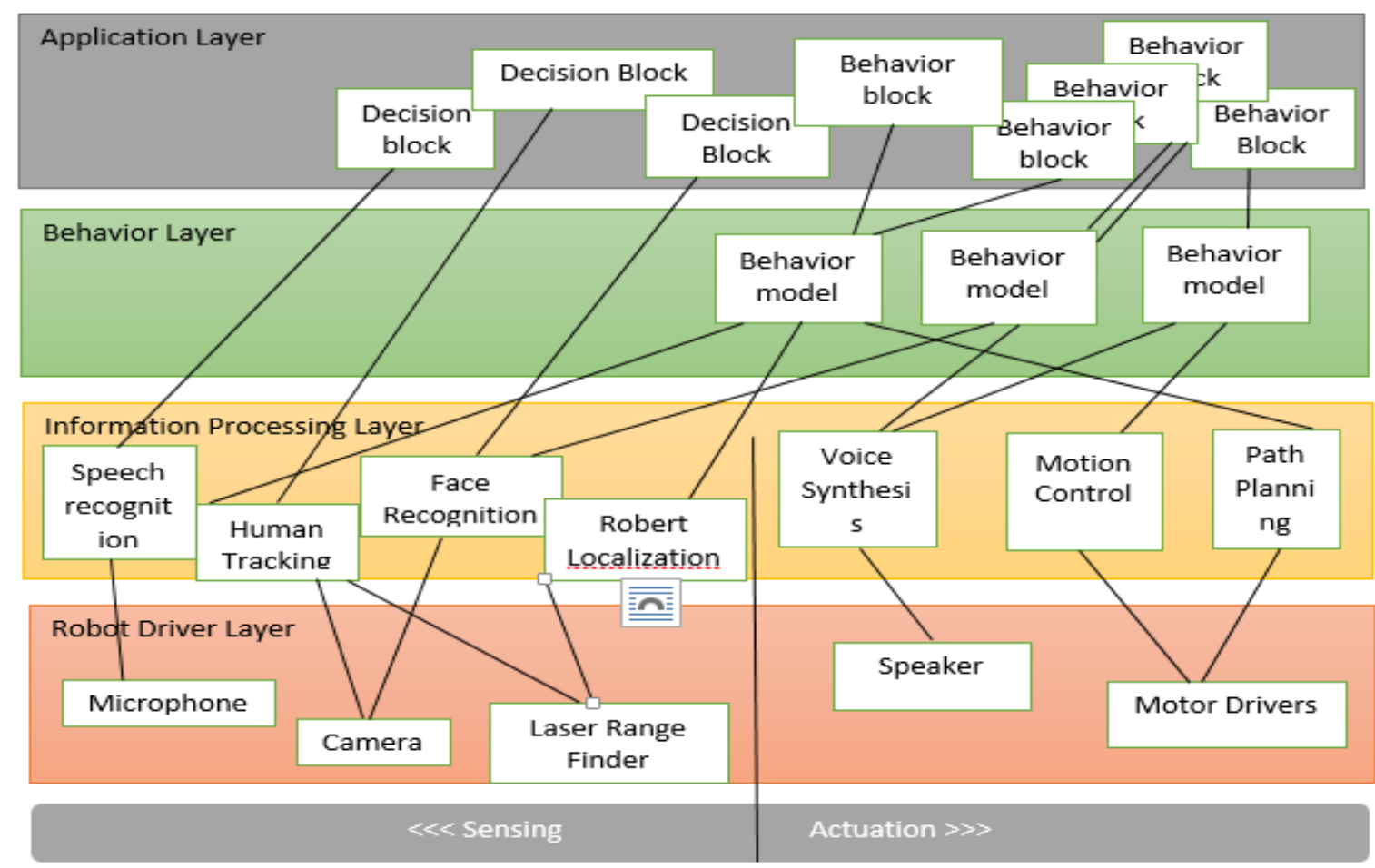

Figure2: Four-layer robot control architecture.

\section{INTERACTION DESIGN FRAMEWORK}

The heading of a section should be in Times New Roman 12point bold in all-capitals flush left with an additional 6-points of white space above the section head. Sections and subsequent sub- sections should be numbered and flush left. For a section head and a subsection head together (such as Section 3 and subsection 3.1), use no additional space above the subsection head.

\subsection{Division of Roles:}

Categorization the main developers of a robot application into "programmers" and "designers."

\subsubsection{Programmer}

There are several tasks which requires programmer to be expertise.

Hardware interfacing: Adding new sensors or actuators to the system to enable the new components to operate with the robot's control system.

Data processing: New recognition techniques or machine learning algorithms will be necessary to help the robot understand the situation in its environment.

Behaviour development: Basic interactive robot behaviours need to be developed.

\subsubsection{Interaction Designer}

The tasks of an interaction designer is centred around the creation of content for the robot's interactions, and the creation of logical sequences of robot behaviours to be executed. The design tasks include the following:

Dialogue generation: An interaction designer will need to specify the robot's gestures. To tune the robot's performance, a designer could adjust the speed of the robot's actions or speech.

Interaction flow design: Interaction flow can be created by linking the robot's behaviors, a designer can create interactions flows. Designer has to consider the order in which the robot presents the information, when to ask questions and how it should respond to a person's actions.

Content entry: It may also be necessary to enter large amounts of domain-specific content, such as items in a restaurant menu, details about products in a store, directions to locations in a shopping mall, etc.,

\subsection{Robot control architecture}

This system uses four layered architecture

\subsubsection{Robot Driver Layer}

The lowest layer is the robot driver layer, which contains hardware-specific driver modules. These modules support abstract interfaces that hide minor differences between similar robots. This enables the same applications and behaviours to 
be used with different robots, as long as they are functionally similar.

\subsubsection{Information Processing Layer}

The information processing layer contains sensing and actuation modules. Sensing modules are components related to recognition of environments and activities in the real world. Localization, human tracking, face detection, speech recognition, and sound source localization are few examples Actuation modules perform processing for tasks like path planning. Some frameworks (e.g. Microsoft RDS), are primarily targeted towards development at this level for robotics research or education, but our framework considers this layer mainly as infrastructure to enable the creation of higher-level behaviors.

\subsubsection{Behavior Layer}

The concept of a robot "behavior" as a combination of sensor processing and actuation is used both in behavioral robotics and in social robotics. In our architecture, behaviors are implemented as software modules in the behavior layer which execute actions and react to sensor inputs. They can incorporate social knowledge. It is also possible to design behavior modules to be configured by designers from the application layer. This is a powerful concept, as it enables the development of flexible, reusable behavior modules.

\subsubsection{Application Layer}

The highest layer is the application layer, where designers can develop social robot applications. This software enables interaction flows to be built by assembling behavior and decision blocks into sequences resembling flowcharts.

\section{EXAMPLES OF SOCIAL ROBOTS}

AIBO: can speak and understand speech, it is able to walk and to recognize objects and people.

iCat: can speak and understand speech, perceive and recognize people and objects in its environment, it can show facial expressions and reacts to touch, iCat is not mobile.

BIRON: can speak and understand speech, recognize people and objects in its environment, it can remember the position of objects and people in a room, drive, and display information on its screen.

BARTHOC: can speak and understand speech, it can show facial expressions and gestures, perceive and recognize people and objects in its environment, BARTHOC is not mobile.

\section{ADVANTAGES AND DISADVANTAGES}

\subsection{Advantages}

Speed: Robots can be significantly faster than people too.

Size: Robots can come in any size. Whatever size needed for any task can be created.

Environment: Robots can be designed to work in extremely harsh environments, such as in space, without air, underwater, fire, etc. Thus, robots can be used instead of people when human safety is a concern.

Dangerous and Unwanted Work: Finally, robots can do jobs that people are unwilling to do. For instance, many robotic probes have been sent throughout the solar system to never return back to Earth. I don't think many people would be willing to do those types of jobs.

Warfare: Using robots in warfare eliminates putting more people at risk and has proven to be very successful.

\subsection{Disadvantages}

Jobs: In my opinion, the biggest issue with using robots is the huge loss of jobs for people. Basically, robots have eradicated a wide range of middle class jobs in several industries, such as car manufacturing.

Limited Functionality: Robots are very good at doing perfectly defined jobs, however robots typically do not handle the unexpected as well as people do.

Lack of Intelligence: Since robots are not intelligent or sentient, robots can never improve the results of their jobs outside of their predefined programming. In other words, robots do not think. At least, not yet.

Lack of Emotions or Conscience: Similarly, robots do not have emotions or conscience; therefore, robots lack empathy and all of the advantages that come with it. As a result, this limits how robots can help and interact with people.

\section{CONCLUSION}

This study suggests the use of NLP to extract personal information, hobbies and interests associate with the user and use it to build a user profile for each new user or update a user profile of an old user whenever new information is extracted. The information from the user profile is used to customize and adapt the dialogue system to fulfil user needs, show empathy and friendship between the robot and the user to foster longterm interaction. Social robots are useful for Children as they are more interested in experiencing new technology and for elderly people. Personal robots are well accepted product and therefore it can be employed to work with/as humans. As a future work, a similarity measure will be used to find connections between various user profiles stored in order to create a family or friendship tree and provide better understanding of the user.

\section{REFERENCES}

[1] I.A. Hameed et al. "User Acceptance of social Robots"In Proceedings of Ninth International Conference on Advance in Computer-Human Interactions, Venice, Italy, pp. 274-279,2016.

[2] C. Breazeal, "Role of expressive behaviour for robots that learn from people" Philosophical Transactions of the Royal Society B: Biological Sciences, 364 (1535), 3527-3538, 2009.

[3] M. Lohse, F. Hegel and B. Wrede, "Domestic Applications for Social Robots - an online survey on the influence of appearance and capabilities" Journal of Physical Agents, vol. 2, no. 2, pp. 21-32, 2008.

[4] Zukerman and D. Litman, "Natural Language Processing and User Modelling: Synergies and Limitations" User Modelling and User-Adapted Interaction, vol. 11, no. 1, pp.129-158, 2001. 